\title{
Effects of shear and walls on the diffusion of colloids in microchannels
}

\author{
S. Ghosh, F. Mugele and M.H.G. Duits* \\ Physics of Complex Fluids group, Department of Science and Technology, \\ MESA $^{+}$institute, University of Twente \\ PO Box 217, 7500 AE Enschede, The Netherlands \\ *Email: m.h.g.duits@utwente.nl
}

\begin{abstract}
Colloidal suspensions flowing through microchannels were studied for the effects of both shear flow and the proximity of walls on the particles' self-diffusion. Use of hydrostatic pressure to pump micron-sized silica spheres dispersed in water/glycerol through poly (dimethylsiloxane) channels with a cross section of $30 \mu \mathrm{m} \times 24 \mu \mathrm{m}$, allowed variation of the local Peclet number ( $\mathrm{Pe}$ ) from 0.01 to 50 . To obtain the diffusion coefficients, imagetime series from a Confocal Scanning Laser Microscope were analysed with a method that, after finding particle trajectories, subtracts the instantaneous advective displacements and subsequently measures the slopes of the Mean Squared Displacement in the flow $(x)$ and shear $(y)$ directions. For dilute suspensions, the thus obtained $D_{x}$ and $D_{y}$ are close to the free diffusion coefficient, at all shear rates. In concentrated suspensions, a clear increase with Peclet number (for Pe>10) is found, that is stronger for $D_{x}$ than for $D_{y}$. This effect of shear-induced collisions is counteracted by the contribution of walls, which cause a strong local reduction in $D_{x}$ and $D_{y}$.
\end{abstract}

Keywords: diffusion, colloids, shear flow, confinement, microfluidics, particle tracking

\section{INTRODUCTION}

Understanding the diffusion of colloidal particles in micro channel flows is important for both fundamental and practical reasons. Shear flows can be strong enough to cause anisotropy in the diffusion coefficient, which in turn can lead to structural inhomogeneity or ordering ${ }^{1-6}$. Additionally, the confining walls which are required to set up the flow, have their own influence on the particle dynamics. Especially how the combination of the two effects works out is far from understood, as most research efforts into (anomalous) diffusion have been focused on either the shear flow ${ }^{7-18}$ or the wall-confinement at rest ${ }^{19-}$ 27.

Fundamental studies of shear-induced diffusion at low volume fraction (where particle interactions are generally weak) were done for both Brownian ${ }^{28-30}$ and non-Brownian ${ }^{10,17,18}$ suspensions. Studies on dense colloidal suspensions are scarcer. In two recent papers addressing this regime, string formation in simple shear flow was observed ${ }^{1,2}$ and explained from anisotropy in the diffusion coefficient. Possible implications hereof for directionally dependent colloidal interactions were mentioned. Earlier, also shear-induced 
migration in concentrated suspensions was observed ${ }^{16,31-34}$ and explained using anisotropic diffusion. Stokesian Dynamics simulations ${ }^{35,36}$ have significantly contributed to the understanding of diffusion in concentrated systems by taking into account both the structural and hydrodynamic effects.

The current scarceness of investigations into shear-induced diffusion of Brownian suspensions ${ }^{1,8,28,29}$ could partly be ascribed to difficulties in measuring diffusive behavior that is superimposed onto a flow. This applies in particular to flows at high Peclet number, where relatively small thermal motions are superimposed onto large advective displacements. But also the complexity of the phenomenon may have hampered studies. Phenomena like anisotropic diffusion and structure formation have shown a rich behavior even for simple (e.g. hard sphere) systems in well-defined (e.g. simple shear) flows s $^{37,38}$. Surprisingly, ordering of particles has been observed only in oscillatory shear flow and the reserchers ${ }^{3}$ could not find any evidence of structuring in steady shear field. To what extent these phenomena could also be influenced by gradients in shear rate, is unclear at present. In pressure-driven channel flows, the shear deformation patterns are different than in Couette or parallel plate flows; this might have consequences for the spatial distribution of the particles and/or ordering in the fluid. A recent study ${ }^{39}$ on charged colloids in electro-osmotically induced flow showed that the average diffusivity (in the flow direction) increases with flow velocity in strongly interacting systems, but not in weakly interacting or dilute systems. The spatial distribution of the diffusivity might have played a role but was not accessible.

Confining walls are expected to play a role, especially in narrow microscopic geometries where they occur in close proximity to all particles. But the spatial extent of their influence is only well-known for a single particle near a single wall, in absence of flow. Only few studies have been focused on diffusion in concentrated systems near walls $s^{20,21,23}$. The interplay between the effects of shear and wall on the diffusion has hitherto never been addressed (as far as we know).

From an applied perspective, knowledge about the diffusive behavior in flow is important to understand and design the mixing of particles, the spreading of a liquid plug, or the formation of concentration gradients perpendicular to the flow direction. This applies in particular to microfluidics, where colloids are used for various purposes. Most current applications concern dilute systems using particles as tracers or scavengers ${ }^{40,41}$, but the flow of concentrated colloidal fluids through micro channels (e.g. in filtration, or after onchip synthesis) is emerging. Other practical scenarios where the diffusive behavior of particles plays a role are drug delivery ${ }^{42}$, the operation of semi-solid flow batteries ${ }^{43}$, the handling of nuclear waste ${ }^{44}$, and stagnant or slowly moving slurries of clay or sand particles in geological rock formations ${ }^{45}$. 
Based on the foregoing considerations, it is clear that still a lot of understanding needs to be gained about how the diffusive behaviour of Brownian particles is influenced by the magnitude of the shear rate, its spatial variation and the proximity of the wall. In this work, we consider the diffusion coefficient (in two directions) of nearly hard sphere colloids in a Poiseuille-like flow, generated by pressure drop over a microfluidic channel. Use of rectangular flow geometry allows considering local flow patterns that are effectively 2-dimensional; the effects of shear and wall can then be studied in a single plane. Due to the lateral variation of the flow velocity, different local shear rates are probed for a given overall flow rate. Repeating the experiment for different pressure drops then allows to achieve the same shear rate at different distances from the wall. This is helpful in separating the contributions of the shear flow (i.e. local Peclet number) and the wall on the diffusion coefficients. We measure the diffusive behaviors by applying particle tracking on data that are collected with a Confocal Scanning Laser Microscope. While this method requires great care to ensure that the advective displacements are adequately removed before the diffusive ones are analyzed ${ }^{46}$, it offers two key advantages: i) the measurements are directional, i.e. both flow and shear directions are examined, and ii) the measurements are spatially resolved, i.e. a distinction is made between the different flow lanes. This makes the method particularly well suited for the study of micron-sized spheres under (nearly) refractive index matched conditions.

As such, it is complementary to other methods: (Confocal) Differential Dynamic Microscopy (DDM) $)^{51-54}$ is able to handle also smaller particles and less transparent fluids, but does not offer positional resolution, and requires prior knowledge of the intermediate scattering function (ISF) ${ }^{52}$ based on the suspension concentration. Without suitable theoretical models for ISF, DDM becomes difficult to especially for concentrated suspensions. Alternatively, Particle Image Velocimetry (PIV) ${ }^{55-56}$ is more suited for measuring local velocities but less so for diffusion. get obscured for a statistically inhomogeneous tracer patterns ${ }^{55}$ or tracer flows coupled with Brownian motion.

We study a low volume fraction $(\Phi=0.03)$ to approach the dilute limit, and an intermediate concentration $(\Phi=0.30)$ to represent a typical situation for transport and mixing of colloids that interact intensely, but not strong enough to cause ordering in the fluid. By comparing these two cases we will inspect the contribution of interparticle collisions to shear-induced diffusion. The scope of addressed Peclet numbers comprises the sub-range of $0.01-50$ for both concentrations, and as such is very well suited to examine both the thermally and hydrodynamically dominated regimes.

\section{EXPERIMENTS}

All experiments were performed at room temperature $\left(22 \pm 1^{\circ} \mathrm{C}\right)$. 


\subsection{Fluid Preparation}

Silica spheres with a $0.5 \mu \mathrm{m}$ diameter core tagged with Fluorescein Isothiocyanate (FITC) and a $1.0 \mu \mathrm{m}$ outer diameter (2a) were synthesized ${ }^{47,48}$ and suspended in water-glycerol ( $1: 4$ by weight) mixtures. With this solvent the refractive index of the particles is nearly matched (close enough for confocal microscopy) while the viscosity $\eta \sim 100 \pm 5 \mathrm{~Pa} \cdot \mathrm{s}$. The fluorescence of the particles was found not to degrade even after years of storage. Also the colloidal stability was preserved over this period, in spite of the (omnipresent but weak) van der Waals attractions. This implies that the particles carry some weak surface charge. Inspection of suspensions with optical microscopy did not show any evidence for long range ordering (see movie 1 ). This suggests that the repulsions are only significant at short-ranges (see Appendix A2), and hence the particles should show a 'nearly' hard sphere behavior.

Volume fractions of 0.03 and 0.3 were achieved by mixing weighed amounts. Measuring the mass density and the 'dry weight' fraction of a silica stock dispersion in pure water, and assuming no excess mixing volumes gave a mass density of $1.89 \mathrm{~g} / \mathrm{ml}$ for the silica. Using the solvent mass density of $1.20 \mathrm{~g} / \mathrm{ml}$, we then calculated how much waterglycerol mixture was needed to redisperse the silica present in a metered amount of stock. Solvent transfer was then achieved by a 4 times repeated centrifugation and resuspension.

\subsection{Microfluidics and Microscopy}

We used $2 \mathrm{~cm}$ long PDMS microchannels with rectangular cross-sections (Fig. 1a). The channel design was fabricated in SU8 by lithography, replicated in PDMS, and bonded onto a $170 \mu \mathrm{m}$ thick glass coverslip. Teflon tubing $(0.91 \mathrm{~mm}$ inner diameter) was used to connect the inlet and outlet of the channel to elevated reservoirs with the colloidal suspension and the solvent, respectively. To facilitate the filling of the channel, it was first flushed with pure solvent. Subsequently the hydrostatic pressure drop was reversed to let the suspension flow in. After the particles had reached the other end, the pressure difference was set to zero and 10 minutes were waited to allow homogenization in the $Y$ and $Z$ directions. The flow rate inside the channel was tuned by adjusting (with sub-mm accuracy) the height difference between the fluid columns. The lowest centreline velocity that could thus be reached was of $\mathrm{O}(0.01 \mu \mathrm{m} / \mathrm{s})$. The highest explored velocity was 10 $\mu \mathrm{m} / \mathrm{s}$, which was still low enough to avoid formation of a concentration peak at the channel centre ${ }^{31,32}$. After adjusting the pressure head (typically 10 values were explored per experiment) 1 minute was given to let the flow become steady again. Observations were made at a distance of $\sim 1 \mathrm{~cm}\left(10^{4}\right.$ particle diameters) from the channel inlet to avoid the entry length effect ${ }^{31}$, and at the symmetry plane $12 \mu \mathrm{m}$ above the bottom to eliminate vertical shear components. 


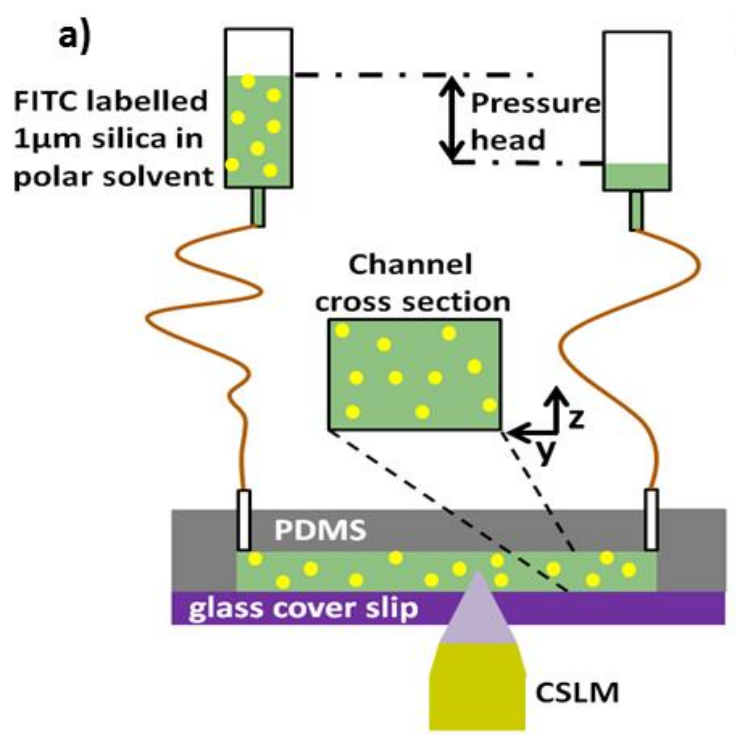

b)

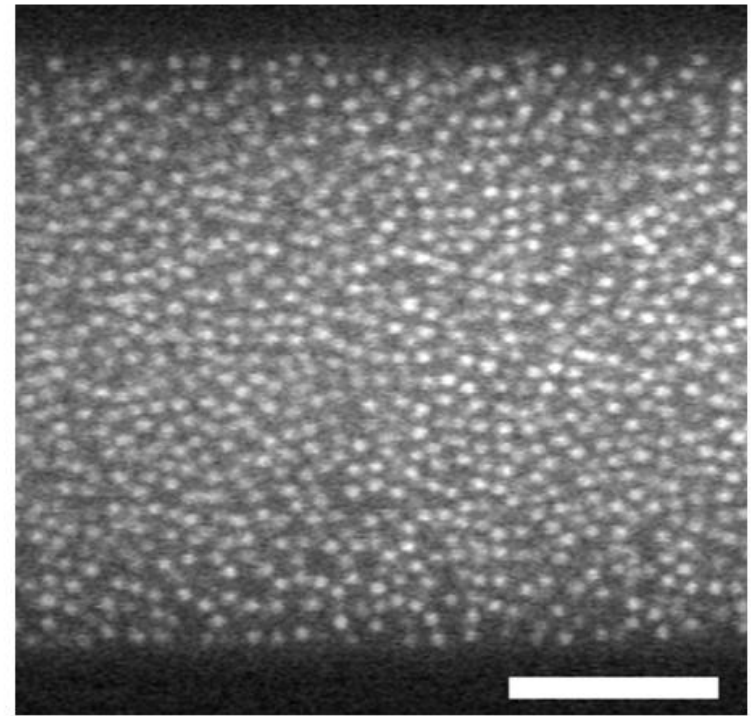

Fig.1 (color online): a) Schematic of the experimental setup. Typical $(X, Y, Z)$ channel dimensions are $2 \mathrm{~cm}, 30 \mu \mathrm{m}$ and $24 \mu \mathrm{m}$. b) Confocal image taken at a height of $12 \mu \mathrm{m}$ from the bottom of a suspension at volume fraction 0.3 , flowing through the channel from left to right with a maximum velocity of $5.4 \mu \mathrm{m} / \mathrm{s}$. Scale bar: $10 \mu \mathrm{m}$.

Images (Fig. 1b) were obtained with an UltraView LCI10 Confocal Scanning Laser Microscope (CSLM) in fluorescence mode, using a 488nm laser, and a 100X/NA1.3 oil objective giving a field of $(X, Y)$ view of $88 \times 67 \mu \mathrm{m}^{2}$ and an effective pixel size of $0.135 \times 0.135$ $\mu \mathrm{m}^{2}$. Most data were collected at a rate of $10 \mathrm{fps}$ using a Hamamatsu 12 bit CCD camera. Typically, 500 frames were grabbed at $\Phi=0.3$ while it was 1500 frames at $\Phi=0.03$. Additionally, a few movies of the low volume fraction sample were collected at the same magnification and frame rates up to $100 \mathrm{fps}$ using a Visitech Infinity-3 system equipped with a Hamamatsu (flash 4.0) camera.

Accurate localization (along $Z$ ) of the $X, Y$ plane where the flow velocities are maximal, is important for avoiding contributions of velocity gradients in the $Z$ direction. After setting the pressure drop, this $Z$ location was determined visually by moving the objective using the piezo positioner and judging the (changes in) flow speed. To allow a posteriori verification, time series were recorded at different Z-locations. Our data analysis (as explained in Sec. 2.3) corroborated that the velocity profile in the vertical direction had the expected shape, and that the optimal Z- location was always very close to the midplane of the channel.

\subsection{Data analysis}

To measure the velocity profile we extended publicly available particle tracking $\operatorname{codes}^{49,50}$, to allow accurate measurement of diffusive motions that are superimposed onto a flow ${ }^{46}$. Briefly, the flow velocity $v_{x}(y)$ is first measured by dividing the $Y$-range into bins and 
averaging intra-bin displacements over (many) frames. Using an iterative scheme, subtraction of the (estimated) advective displacements is used to improve the quality of the tracking per iteration step. The diffusive motions are obtained from the trajectories of the final iteration, by first subtracting the instantaneous advective displacements (this eliminates the effect of Taylor dispersion ${ }^{8,57}$ ), subsequently calculating the Mean Squared Displacements in the $x$ - and $y$-directions, and finally by fitting these obtained (lag-time dependent) MSDs to a straight line in order to obtain $D_{x}(y)$ and $D_{y}(y)$. The measured diffusion coefficients are short-time self-diffusion as the involved time scale $(O(10))$ is much smaller than the long-time $(\mathrm{O}(100))$ measurements.

From the velocity profile $v_{x}(y)$, the local shear rate is determined by taking the gradient: $\dot{\gamma}(y)=\frac{d v_{x}}{d y}$, and subsequently converted to the dimensionless Peclet number (Pe), defined as the ratio between the Brownian diffusion time $\left(\tau_{B}=\mathrm{a}^{2} / D_{0}\right)$ and the advective diffusion time $\left(\tau_{C}=\dot{\gamma}^{-1}\right)^{31,32}$ :

$$
P e=\frac{\tau_{B}}{\tau_{C}}=\frac{a^{3} \dot{\gamma}(y)}{D_{0}}
$$

where $a$ is particle radius and $D_{0}$ is its free diffusivity in the dilute limit.

\section{RESULTS AND DISCUSSION}

\subsection{Velocity profiles}

The velocity profiles measured at different pressure drops are illustrated in Fig. 2a for $\Phi=0.03$ and in Fig. $2 b$ for $\Phi=0.3$. They appear smooth for all concentrations and pressure drops. The highest local velocity is $10 \mu \mathrm{m} / \mathrm{s}$. Since this produces elongated images of the particle for exposure times of $100 \mathrm{~ms}$, some additional experiments were performed with a high-speed confocal system (section 2.3). At the lowest flow rate, the maximum velocity is only $0.01 \mu \mathrm{m} / \mathrm{s}$. The smoothness and near-parabolic shapes of the velocity profiles indicates that they were accurately measured.

This is further corroborated by analysing the mechanics of the flow problem. For Newtonian fluids, the amplitude of $v_{x}(y)$ should be proportional to $\Delta P$, while its shape should remain constant. The former turns out to be the case within the measurement accuracy of the pressure drop. In Figs. $2 c$ and $d$ we inspect the flow-rate dependence of the shape of $v_{x}(y)$ by normalizing each curve via its maximum. The changes in shape turn out to be small. Due to the way of normalizing, the largest deviations are seen near the walls, where velocities are lowest. This is best visible for $\Phi=0.03$ (Fig. 2c). However, at this volume fraction the suspension should behave as a Newtonian liquid at all shear rates. The deviations at low velocities might be due to ( $x-)$ drift of the microscope table, which gets incorporated in the measured $v_{x}(y)$ as an offset. Usually in vibration isolated systems, a microscope table will always show a slow motion with respect to the objective 
(noise). This motion gets incorporated in the found trajectories of the particle. In experiments without flow, a correction for this drift is possible by tracking the center of mass of all particles. In flow experiments, the motion contains both the flow velocity and the table drift velocity. These two are difficult to separate, but generally the drift is negligible: except at very low local velocities.

In Figs. 2c and d, also a comparison is made with theoretical profiles for a Newtonian liquid in the given channel geometry. Assuming no-slip boundary conditions at the walls, the velocities can be expressed $a s^{58}$ :

$$
v_{x}(y, z)=\frac{4 h^{2} \Delta P}{\pi^{3} \eta_{0} L} \sum_{n, o d d}^{\infty} \frac{1}{n^{3}}\left[1-\frac{\cosh \left(n \pi \frac{y}{h}\right)}{\cosh \left(n \pi \frac{w}{2 h}\right)}\right] \sin \left(n \pi \frac{z}{h}\right)
$$

with $\mathrm{x}, \mathrm{y}$ and $\mathrm{z}$ and $\Delta P$ as previously defined, $h, w$ and $L$ the channel height, width and length, and $\eta_{0}$ the viscosity. The agreement between experiments and theory appears to be good. Small deviations near the walls might be caused by the finite size of the particles in the experiments: in reality the particles also need to rotate, to accommodate the velocity gradient. This effect could change the flow pattern somewhat, especially close to the walls. The Newtonian velocity profile also appears to describe the experiments at $\Phi=0.3$ well. In principle, flow curves of colloidal hard spheres should show a transition from a low- to a high-shear plateau around $P e \approx 1$. Measurement of the viscosity of a similar (near)hard sphere suspension (water-glycerol mixture with $0.96 \mu \mathrm{m}$ silica spheres at $\Phi=0.34$ ) by $X$. Cheng et $a l .{ }^{59}$ for different shear rates, clearly shows the Newtonian nature of the suspension between Pe numbers 3 and 110 . Summarizing, the deviations from the theoretical velocity profiles for Newtonian fluids are modest, and do not suggest that there are issues regarding the correctness of the measured particle velocities ${ }^{60-62}$. 

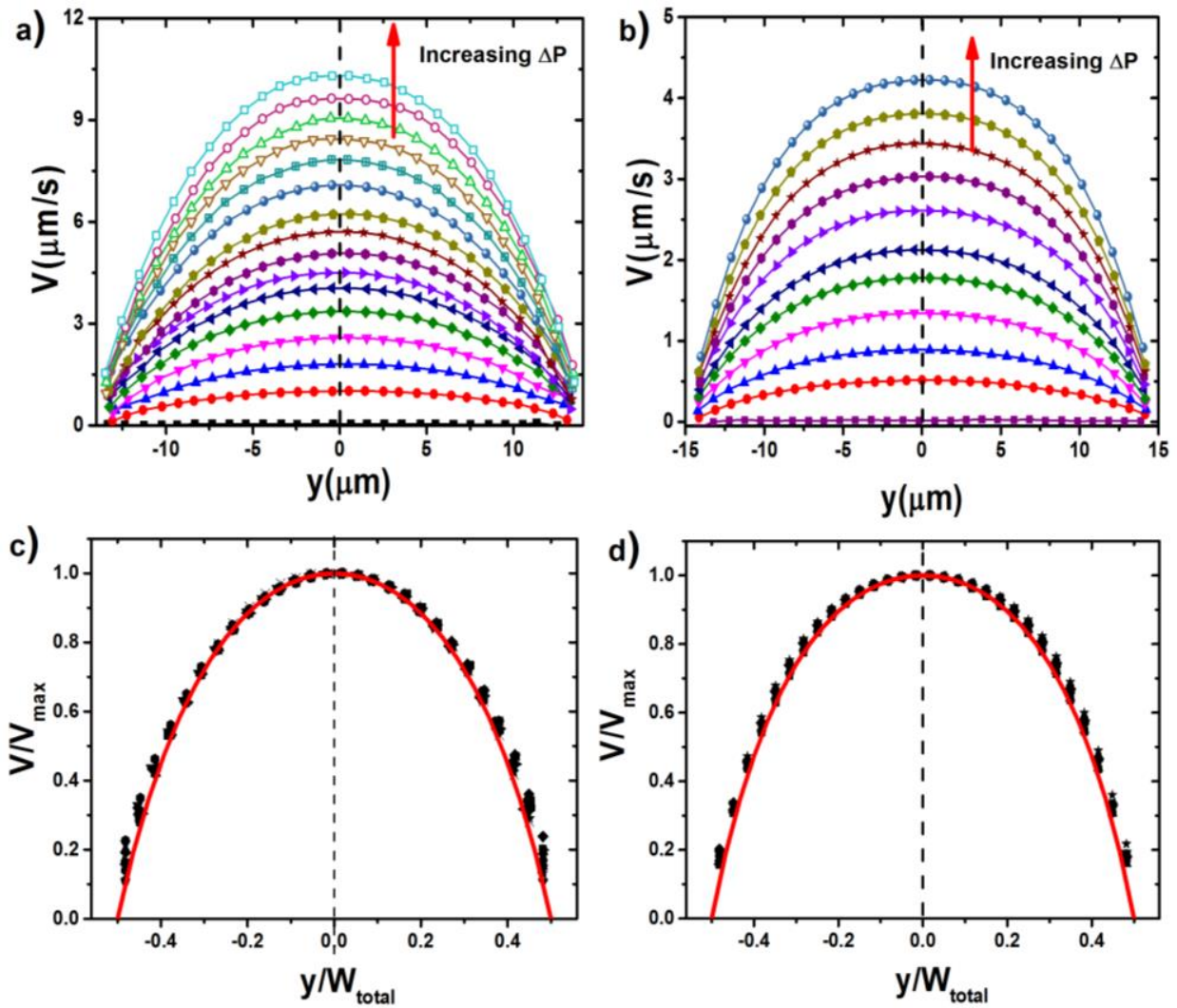

Fig. 2 (color online): Particle velocity profiles across the channel for different pressure drops. Panels a) and b) show the measurements from the videos. Panels c) and d) show the comparison of normalized velocity profiles (black points) with theory (red lines). Particle volume fractions are 0.03 for a) and c), and 0.3 for $b$ ) and d).

\subsection{Diffusion Coefficients}
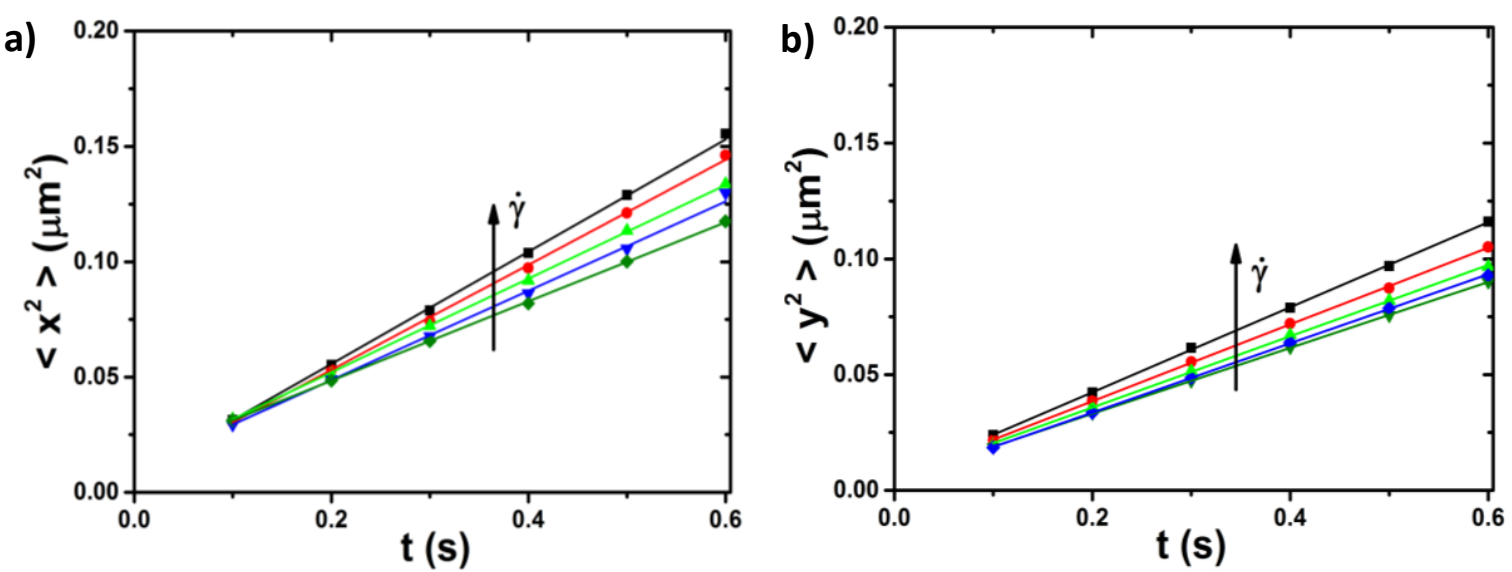

Fig. 3 (color online): Mean Squared Displacements (MSD) for a suspension at $\Phi=0.3$, for the experiment where $v_{\max }=5.4 \mu \mathrm{m} / \mathrm{s}$. Different datasets in the same panel correspond to different locations: from bottom to top $y=4.5,5.6,7.5,9.7$ and $10.5 \mu \mathrm{m}$ from the centre line. This order corresponds to an increasing local shear rate $(\dot{\gamma})$. Panel a): MSDs in flow direction (after correction for advection) and panel $b$ ): MSDs in the velocity gradient direction. Solid lines are linear fits to the experimental data. 
Typical MSDs obtained for an experiment at $\Phi=0.3$ are shown in Fig. 3 for both flow (Fig. 3a) and shear (Fig. 3b) directions. The linearity of the data is good, also for the flow direction where (large) advective displacements had to be subtracted first. Both MSDs are found to depend on $y$-location; this is ascribed to the $y$-dependence of the local shear rate. Extrapolation of the linear fits to zero lag-time reveals that both MSDs have an offset of $\approx 70 \mathrm{~nm}^{2}$. This value is close to the typical noise floor of an MSD measurement ${ }^{27}$, but still contributes significantly to the magnitudes of the MSDs. Therefore $D_{x}$ and $D_{y}$ are calculated from the slopes of the linear fits to the MSDs (using the Einstein relation).

\subsubsection{Influence of shear}

In dilute suspensions $(\Phi=0.03)$, both $D_{x}$ and $D_{y}$ are practically equal to each other, and almost independent of the overall flow rate and the $y$-location (data shown elsewhere ${ }^{46}$ ) Exceptions to this are only found in close proximity of the walls due to the (anisotropic) hydrodynamic resistance close to the wall. As the particles are only weakly interacting with each other in dilute suspensions, their diffusive behavior should be similar to that of a solitary particle; at rest as well as in flow. In contrast, for concentrated systems $(\Phi=0.3)$, both $D_{x}$ and $D_{y}$ do show a dependence on both overall flow rate and $y$-location. Representative examples are shown in Fig. 4 for a low and a high flow rate.
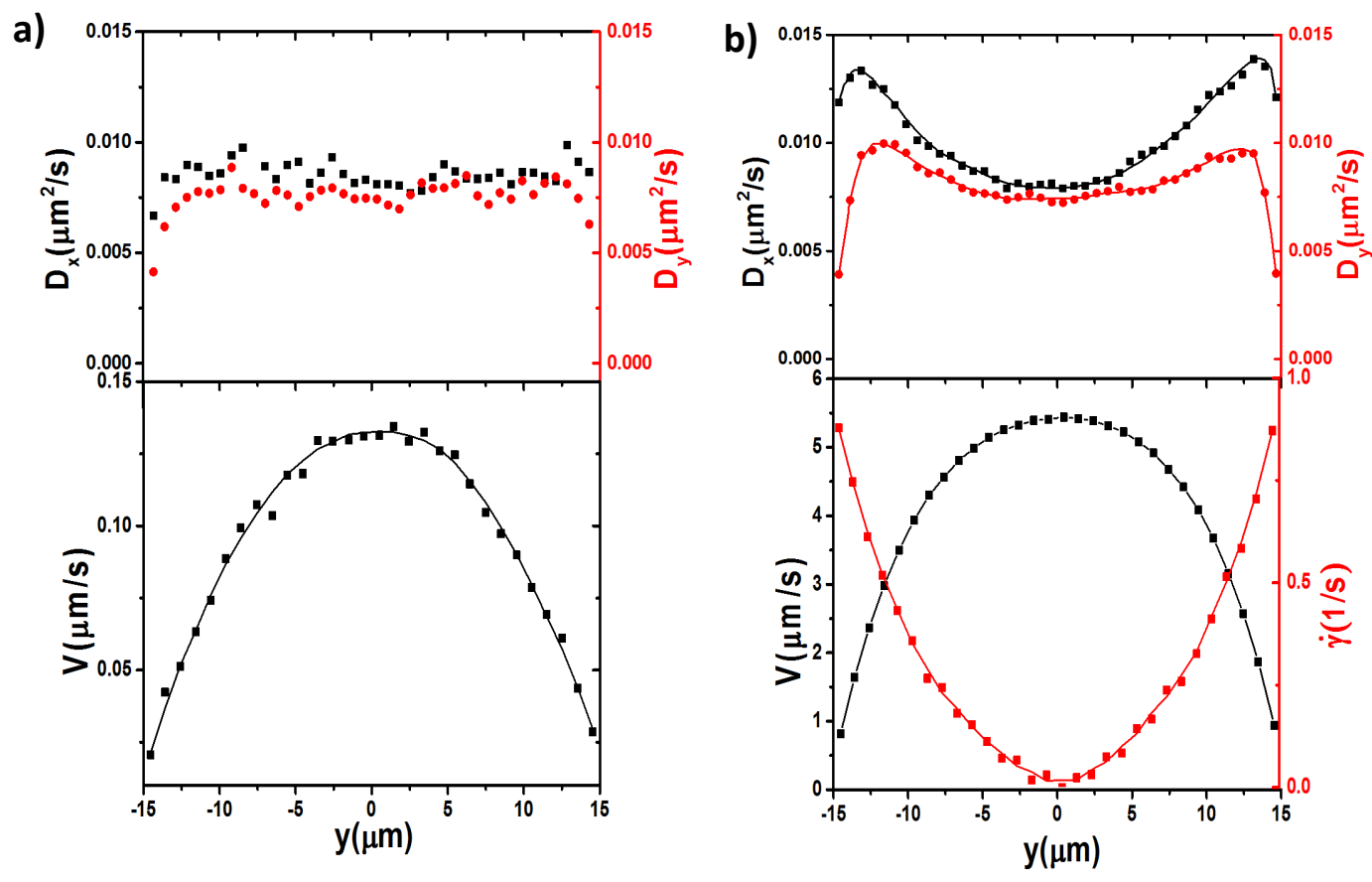

Fig.4 (color online). Experimental results for a concentrated suspension ( $\Phi=0.3)$ nearly at rest (a) and in a strong flow (b). Upper graph: (black square) diffusion coefficients in the flow $(x)$ and (red circle) shear $(y)$ directions as a function of the lateral $(y)$ position in the channel. Lower graph: Local velocity and shear rate in the same channel. The walls are located at $y= \pm 15 \mu \mathrm{m}$. Symbols represent experimental data while lines are to guide the eye. 
Analysing $\mathrm{D}_{\mathrm{x}}(\mathrm{y})$ and $\mathrm{D}_{\mathrm{y}}(\mathrm{y})$ in conjunction with the local shear rate $\dot{\gamma}(y)$ reveals that both diffusion coefficients are influenced by two opposing effects; $i$ ) first of all, the diffusion is enhanced by the local shear. At the centreline $\mathrm{y}=0$ where $\dot{\gamma}$ is zero, $\mathrm{D}_{\mathrm{x}}$ and $\mathrm{D}_{\mathrm{y}}$ are equal while for $|y|>0$ both $D_{x}$ and $D_{y}$ show an (initial) increase. These observations can be attributed to shear-induced collisions, which are well known to occur also for noncolloidal fluids at high concentrations $s^{8,15,16}$. This increase turns out to be stronger for $D_{x}$ than for $D_{y}$. ii) as the side walls are approached, the diffusion coefficients reach a maximum followed by a steep decrease. Considering that the shear rate shows a continuous increase up to the point where the wall is reached, this illustrates that the diminishing effect that the wall has on $D_{x}$ and $D_{y}$, ultimately becomes dominant.

a)

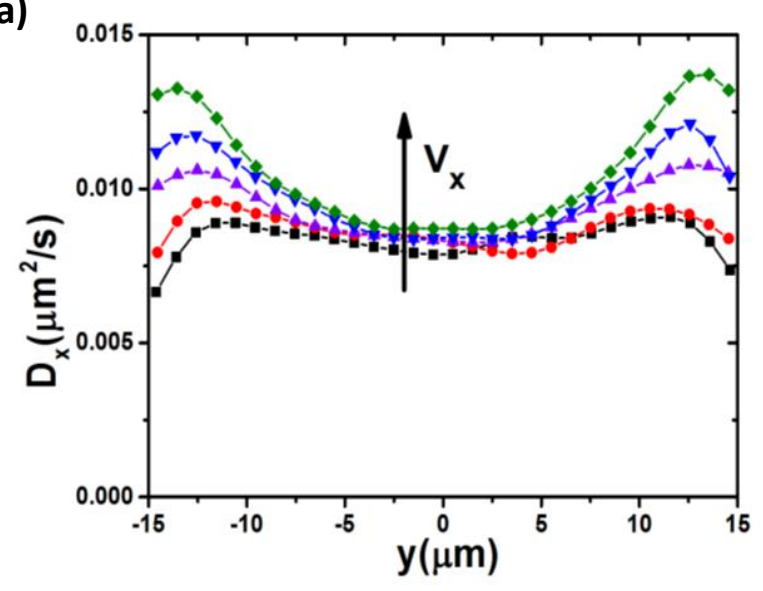

b)

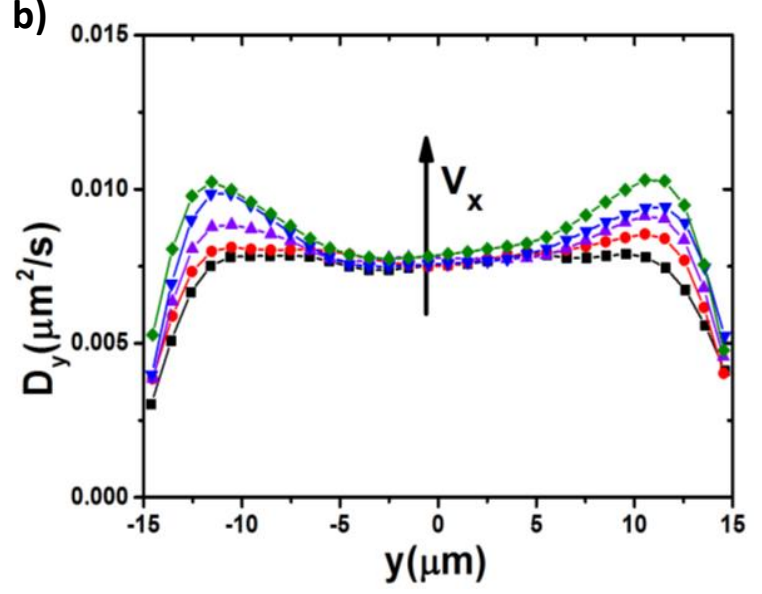

Fig. 5 (color online). Diffusion coefficients in the flow $(x)$ and shear $(y)$ directions as a function of lateral $(y)$ position, for a suspension at $\Phi=0.3$ studied at different flow rates. At $y=0$, fluid elements are advected without any shear (irrespective of flow rates) and all the measured $D_{x}$ and $D_{y}$ coincides. Maximum flow velocities $\left(v_{\max }\right)$ : black squares: 1.44 $\mu \mathrm{m} / \mathrm{s}$, red circles:2.24 $\mu \mathrm{m} / \mathrm{s}$, violet up-triangles:3.05 $\mu \mathrm{m} / \mathrm{s}$, blue down-triangles: 3.89 $\mu \mathrm{m} / \mathrm{s}$, green diamonds: $4.75 \mu \mathrm{m} / \mathrm{s}$. Symbols represent experimental data while lines are to guide the eye.

More insight regarding shear-induced diffusion and the wall effect can be obtained by collecting the results obtained at different pressure drops in the same graph (see Fig. 5). Besides a confirmation of the general trend, this also allows comparing $D_{x}$ and $D_{y}$ at the same $y$-location but different flow rates. Clearly, both $D_{x}$ and $D_{y}$ increase systematically with the flow rate, for all $y$-locations except at the center where $\dot{\gamma}$ is zero regardless of the velocity. This corroborates that both diffusion coefficients are enhanced by shear (to an extent that depends on the shear rate, or as we will see, the Peclet number).

To separate the contributions of the shear flow and the wall, we utilize the fact that many different overall flow rates were explored. As can also be seen from Fig. 2a and b, each curve covers a range of slopes (i.e. shear rates), and the overlap is such that the same $\dot{\gamma}$ can often be found in several different curves: further away from the wall as the flow 
rate gets higher. This allows making graphs of $D_{x}$ and $D_{y}$ vs $y$, under the constraint that the shear rate is the same. Our analysis, most significantly, revealed that (at least) for $|y| \leq 0.7|y|_{\text {max }}$ the data at different velocities superimpose well. This finding, which is in good agreement with the observation that peaks in $D_{x}(y)$ and $D_{y}(y)$ are generally found at $|y| \approx 0.8|y|_{\max }$ (see Figs. 4 and 5), has two implications. Firstly, away from the walls, the diffusion coefficients appear to be determined by the local shear rate only. This makes it possible to construct master plots for $D_{x}$ and $D_{y}$ as a function of $\dot{\gamma}$. And secondly, using these master plots, it should be possible to quantify the wall effect in presence of shear. We first discuss the effect of local shear alone. In Fig. 6 we plot $D_{x}$ and $D_{y}$ as a function of Peclet number. To make the plot more general, diffusion coefficients are normalized with respect to their value in absence of shear. Interestingly, for the dilute system $\left(\Phi=0.03\right.$, Fig. 6a), $D_{x}$ and $D_{y}$ are found to be equal within their uncertainty ranges, for all Peclet numbers. Moreover, a clear dependence on Peclet number is absent. This confirms that under dilute conditions, the motion of a particle is simply a superposition of an unimpaired Brownian diffusion and a spatially dependent advection (in the limiting case, all particle interactions are neglected). Qiu et $\mathrm{al}^{8}$, and Orihara and Takikawa9,28 who used an oscillating flow to study shear-induced diffusion in a very dilute system, observed a constant diffusivity perpendicular to the flow direction as well.

Considering the diffusivity in the flow direction, Orihara et.al. ${ }^{9,28}$ found a strong enhancement by shear. This apparent contrast with our results can be ascribed to the contribution of advection: unlike the earlier studies ${ }^{9,28}$, we have eliminated the local affine motions (in order to highlight the effect of shear induced collisions). Hence both the earlier and our present findings show that the particle motions in flow can be understood from a superposition.
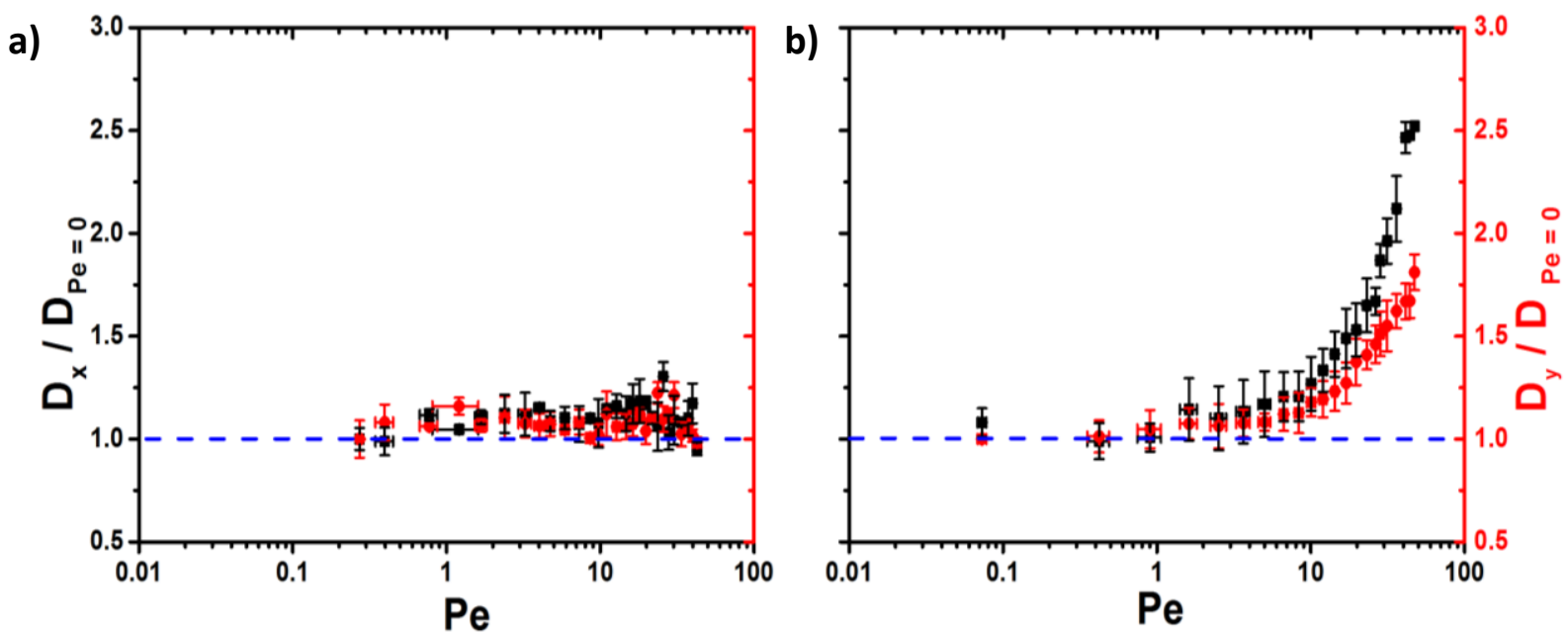

Fig.6(color online). Short time self-diffusion coefficients in the flow $(x)$ and velocity gradient ( $y$ ) directions, as a function of Peclet number (Pe). a) At $\Phi=0.03$, diffusion is isotropic and independent of Pe number. b) At $\Phi=0.3$, the diffusion coefficient is isotropic up to $P e \approx 5$, after which it increases with Pe. Beyond $P e=10$, diffusion becomes strongly 
anisotropic. Red points(circle) are for $D_{y}$ and black points(square) are for $D_{x}$. Blue dashed lines indicate the diffusion coefficient at $P e=0$.

For the concentrated system $(\Phi=0.3)$, the diffusivity of the particle increases with Peclet number (Fig. 6b). This increase applies to both $D_{x}$ and $D_{y}$, and becomes noticeable for $P e$ $>$ 1. A transition from a thermally to a hydrodynamically dominated diffusion regime is indeed expected to take place in this Pe number range. While in this respect our findings are not unexpected, we also like to point out that this is the first time that such a master plot for $D_{x}$ and $D_{y}$ is reported for Brownian (near-hard sphere) suspensions in pressure driven flow. Apparently, the local Peclet number provides adequate specification of the flow, i.e. knowing it suffices to calculate its contribution to the two diffusion coefficients. A striking observation in Fig. $6 \mathrm{~b}$ is that once the shear dominated regime is reached, the diffusion becomes strongly anisotropic. Since all affine motion was taken out prior to calculation of the diffusion coefficients, this trend indicates that that the shear induced collisions have a clear directionality. Superposition of diffusion coefficients (fig.6) from different experiments where the data points correspond to different shear gradients but same Pe number suggests that the gradients in shear rate are not very important for shear induced diffusion.

It is interesting to compare our results to earlier findings. Cheng et. al. ${ }^{1}$, studied a similar fluid in a plate-plate geometry, and found shear enhancement of the diffusion in the velocity and vorticity directions. Remarkably, they used a power law with an exponent of 0.81 to describe the lag-time dependence of the $\mathrm{x}-\mathrm{MSD}$, whereas we found a linear behavior. Considering the effects of spatial confinement ${ }^{27}$ on MSD, this difference might be due to the stronger confinement ( 3 to 10 particle diameters) in their system. In an early study using Stokesian Dynamics, Bossis and Brady ${ }^{13}$ observed a reduction of shorttime self-diffusion coefficients with increasing shear. This opposing trend might be related to the fact that they considered a monolayer of colloids. The enhancement of the $y$-diffusion coefficient as we found, appears in good agreement with the shear induced migration of particles from the wall to the channel centre found by other researchers for concentrated suspensions at high Peclet number ${ }^{31,32}$.

\subsubsection{Combined influence of wall and shear}

Given the master curves for $D_{x}$ and $D_{y}$ as a function of $P e$, it is possible to make a quantification of the contribution of the wall $\Delta D_{x}$ and $\Delta D_{y}$, for different shear rates. We remark that in presence of (strong) flow, the calculated effects are less accurate than the data presented in Fig. 5 and Fig. 6, because the diffusion coefficients become rather small close to the wall and because more data processing steps (see Appendix A1) were needed to arrive at the results. Nevertheless they should still be interesting since they indicate trends that have never been measured. From the master plots (fig.6), we 
extrapolated diffusion coefficients at all shear rates including near the wall. Measuring the difference between extrapolated and observed data for all experiments, we quantified the wall influence on diffusivity.

To assess the accuracy, we first consider the experiment at low volume fraction (0.03). Here the particles can be reasonably approximated as isolated species while the effect of shear is negligible. This renders a comparison of the measured $D_{x}$ and $D_{y}$ with the theoretical expressions meaningful. Approximatively, the reduction in diffusivity relative to the free diffusion coefficient $D_{0}$ can be expressed as:

$$
\beta_{x}^{-1}=\frac{D_{x}}{D_{0}} \cong 1-\frac{9}{16}\left(\frac{a}{\Delta y}\right)+O\left(\frac{a}{\Delta y}\right)^{3}
$$

for diffusion parallel to the wall ${ }^{63-65}$, and

$$
\beta_{y}=\frac{D_{y}}{D_{0}} \cong \frac{6 \Delta y^{2}+2 a \Delta y}{6 \Delta y^{2}+9 a \Delta y+2 a^{2}}
$$

in the perpendicular direction ${ }^{66}$. Here $a$ is the particle radius while $\Delta y$ is the distance between particle centre and wall (note that $\Delta y \geq 0.5$ ). Defining $\Delta D_{x}(\Delta y)$ as $\left[1-\frac{D_{0}-D_{x}(\Delta y)}{D_{0}}\right]$, and similarly for $\Delta D_{y}(\Delta y)$, allows comparison of the experimental data with Eqs. 3 and 4 . The agreement between solid lines and black symbols in Figures 7 a and c turns out to be fairly good; it is the noise in the experimental data which precludes a more accurate comparison.

In the presence of shear flow, we define $\Delta D_{x}$ as $D_{x}(\Delta y)-D_{x}(P e)$, where the latter term is interpolated from the master curve (Fig. 6) after looking up the Peclet number at $\Delta y$. A detailed description of the procedure can be found in the Appendix A1. Fig. 7 shows $\Delta D_{x}(\Delta y)$ and $\Delta D_{y}(\Delta y)$ as function of normalized distance $(\Delta y / d)$ at rest and in flow for $\Phi=$ 0.03 (panels $a$ and $c$ ) and $\Phi=0.3$ (panels $b$ and $d$ ). It is suggested by all graph that, after correcting for the direct effect of shear flow, the remaining deviation in the diffusion coefficient is mainly due to the wall. In other words: indirect effects of the shear flow (e.g. via a change of the local structure) are weak as compared to the local wall effect. This could be expected for $\Phi=0.03$, but also appears to be the case for $\Phi=0.3$.

Two additional remarks can be made: i) for $\Phi=0.3, D_{x}$ is less supressed by the wall as compared to $\Phi=0.03$ (Figs. 7 a and b), and ii) the typical length scale over which $D_{x}$ and $D_{y}$ are influenced by the wall appears to be smaller for the concentrated fluid. This 'increased hydrodynamic screening' is in line with earlier experimental and theoretical measurements ${ }^{23,67}$. 

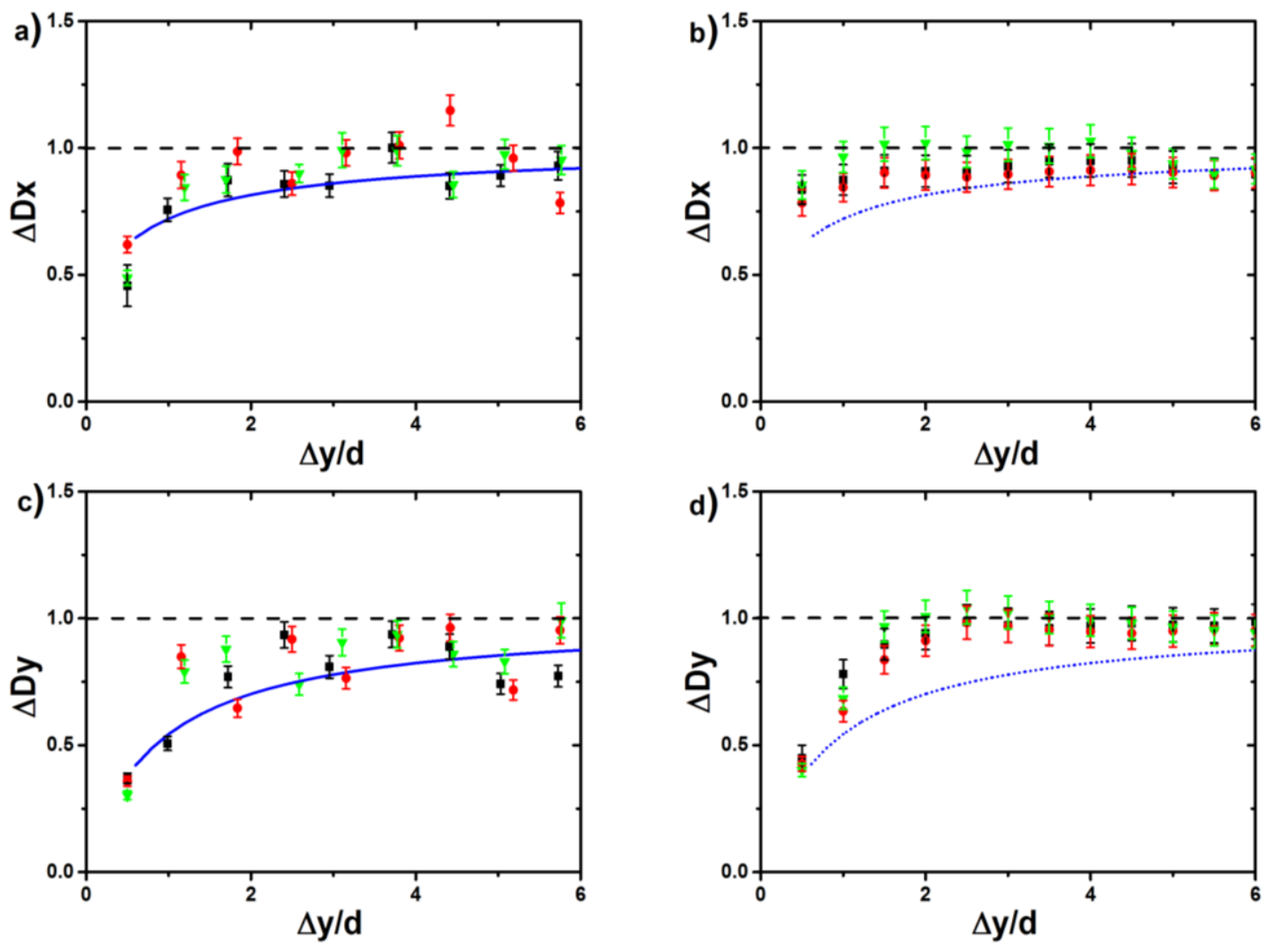

Fig.7 (color online): Change in normalized diffusion coefficients as a function of normalized distance $(\Delta y / d)$ from the wall. $d(=2 a)$ is the particle diameter. Symbols show the experimental data, solid and dotted lines are the analytical solutions (Eqs. 3 and 4) while dashed lines indicate the free diffusion coefficient. Left panels $[a, c]$ show the data for $\Phi=0.03$. Maximum flow speed $\left(v_{\max }\right)$ : black square: $0.16 \mu \mathrm{m} / \mathrm{s}$, red circle: $5.4 \mu \mathrm{m} / \mathrm{s}$, green down-triangles: $10.6 \mu \mathrm{m} / \mathrm{s}$. Right panels $[b, d]$ show the data for $\Phi=0.3$. Here the dotted lines are plotted just to visualize the difference with the experimental data for $\Phi=0.03$. $v_{\text {max }}$ : black square: $0.13 \mu \mathrm{m} / \mathrm{s}$, red circle: $0.80 \mu \mathrm{m} / \mathrm{s}$, green down-triangles: 4.0 $\mu \mathrm{m} / \mathrm{s}$.

\section{CONCLUSIONS}

We studied the shear induced diffusion of dilute and concentrated Brownian near-hard sphere suspensions flowing through micro channels. Direct measurements of such diffusion coefficients in flow are scarce, and our results complement the existing literature. Our measurements indicate that the local Peclet number provides an adequate characterization of the effect of flow on diffusion (except close to the walls). Diffusion coefficients in the flow and velocity-gradient directions show different dependences on $\mathrm{Pe}$ for dilute and concentrated systems. At low volume fraction, both coefficients are equal and practically independent of the shear rate, in line with the definition. At high volume fraction, isotropic Brownian diffusion dominates at low Peclet numbers $(\mathrm{Pe}<1)$ while for $\mathrm{Pe}>>1$ both diffusion coefficients grow, due to shear-induced particle collisions. For Pe $>10$ the diffusion becomes strongly anisotropic. Close to the wall, a strong reduction in diffusivity is observed for all concentrations and shear rates, indicating that as the wall is 
approached, the effect of the wall dominates over the effect of shear. We did not obtain evidence for a strong coupling between the wall and shear effects.

\section{Acknowledgements:}

We thank Daniel Wijnperlé for micro channel fabrication, Dr. Dirk van den Ende for discussions and Dr. Joris Sprakel for allowing us to perform experiments in his lab. This work was financially supported by NWO-CW (ECHO grant 09.FM.016).

\section{Appendix A1}

For a neutrally buoyant particle in Newtonian liquid, the effective diffusion ( $D^{\text {eff }}$ ) is equivalent to the Brownian diffusion $\left(D_{B}\right)$

$$
\begin{aligned}
& D^{\text {eff }}=D_{B} \\
& \frac{D^{\text {eff }}}{D_{B}}=1
\end{aligned}
$$

In presence of a flat wall, the effective diffusion coefficient reduces due to wall influence

$$
\begin{aligned}
& \mathrm{D}^{\text {eff }}=D_{B}+D_{w} \quad\left(D_{w} \text { indicates the influence of wall only }\right) \\
& \frac{D^{\text {eff }}}{D_{B}}=1+\frac{D_{w}}{D_{B}} \quad(A 2.1)
\end{aligned}
$$

This is same as $\mathrm{H}$. Brenner's prediction. Now we assume that the shear effect acts additively with wall effect and hence shear induced diffusivity $\left(D_{\text {sh }}^{\text {eff }}\right)$ can be expressed as

$$
\begin{aligned}
& D_{\text {sh }}^{\text {eff }}=D_{B}+D_{w}+D_{s h}\left(D_{s h}\right. \text { indicates the shear effect only) } \\
& \frac{D_{\text {sh }}^{\text {eff }}}{D_{\mathrm{B}}}=1+\frac{D_{w}}{D_{B}}+\frac{D_{s h}}{D_{B}} \quad(A 3.1)
\end{aligned}
$$

The master curve is constructed considering the data points free from strong wall effect. So the extrapolated diffusivity ( $D_{\mathrm{m}}^{\text {extra }}$ ) from the master curve contains only the shear term which can be formulated by the following way

$$
\begin{aligned}
& D_{m}^{\text {extra }}=D_{B}+D_{s h} \\
& \frac{D_{m}^{\text {extra }}}{D_{B}}=1+\frac{D_{\text {sh }}}{D_{B}}
\end{aligned}
$$

Now, if we subtract the observed diffusivity in shear $\left(D_{\text {sh }}^{\text {eff }}\right.$ ) from the extrapolated diffusivity, we will end up of having the wall effect only and the relation becomes

$$
\begin{aligned}
& D_{m}^{\text {extra }}-D_{\text {sh }}^{\text {eff }}=\left(D_{\mathrm{B}}+D_{s h}\right)-\left(D_{\mathrm{B}}+D_{\mathrm{w}}+D_{\mathrm{sh}}\right)=-D_{\mathrm{w}} \\
& \frac{\mathrm{D}_{\mathrm{m}}^{\text {extra }}-\mathrm{D}_{\mathrm{sh}}^{\text {eff }}}{\mathrm{D}_{\mathrm{B}}}=-\frac{\mathrm{D}_{\mathrm{w}}}{\mathrm{D}_{\mathrm{B}}}
\end{aligned}
$$

If we compare this with Brenner's prediction (comparing eq. A5.1 with eq. A2.1), then the relation becomes

$$
D_{\text {Brenner }}=\frac{D^{\text {eff }}}{D_{B}}=1+\frac{D_{w}}{D_{B}}=1-\frac{D_{m}^{\text {extra }}-D_{\text {sh }}^{\text {eff }}}{D_{B}}
$$

General normalized form can be represented as

$$
\Delta D_{i}=1-\frac{D_{m, i}-D_{i}}{D_{P e=0}} \quad, \quad i=x, \mathrm{y}
$$


where $D_{m, i}$ is the extrapolated diffusivity from master curve. So, for dilute concentration, wall effect on diffusivity at rest as well as in flow can be compared with analytical solution ${ }^{63-66}$.

\section{Appendix A2}

We measured the pair potential of silica spheres (diameter $=1 \mu \mathrm{m})$ in water-glycerol mixtures by approximately measuring the 2-dimensional radial distribution function $(g(r))^{68}$. The potential falls steeply to (near) zero within $\approx 50 \mathrm{~nm}$. This distance seems comparable to other near-HS systems.

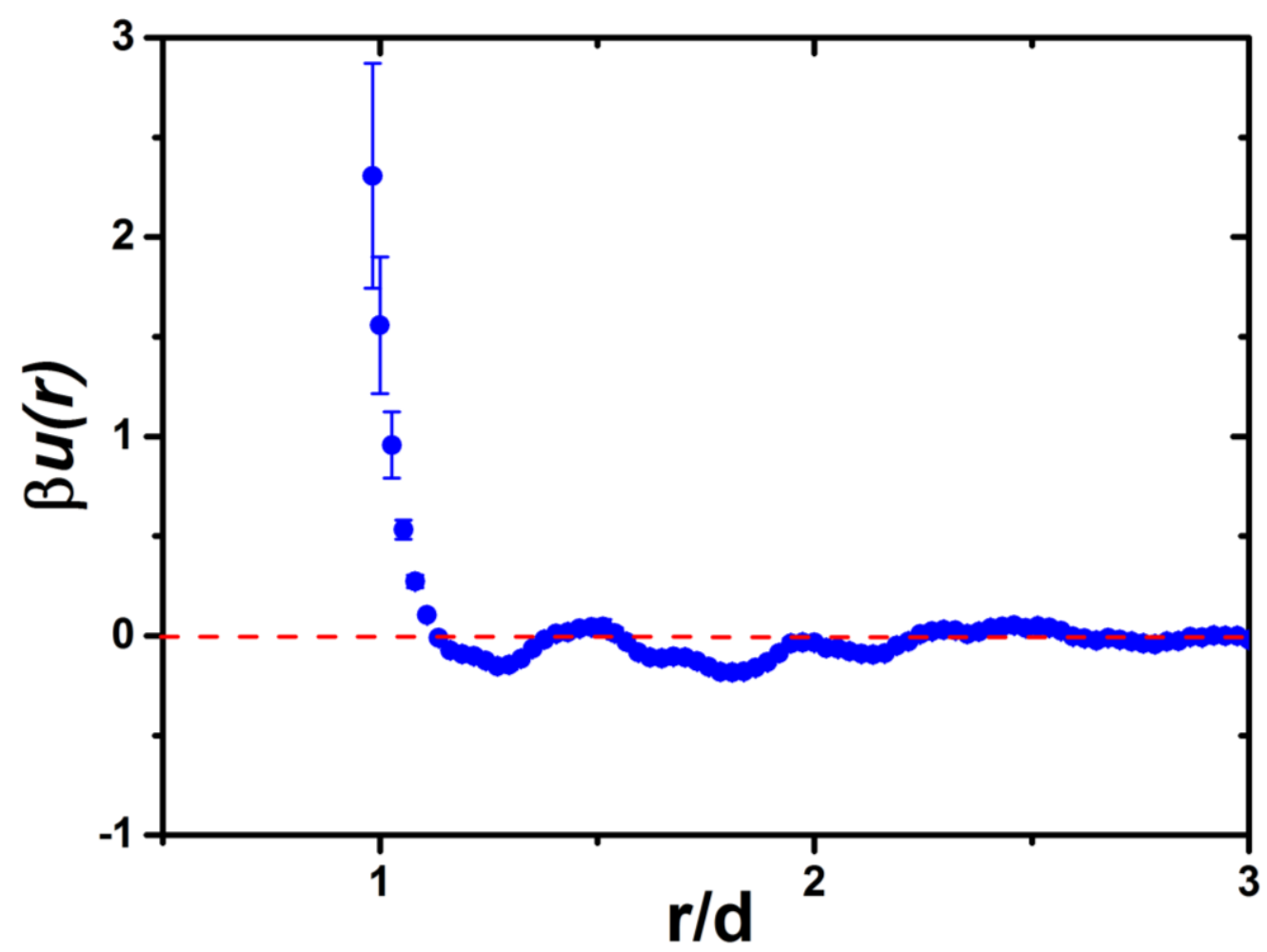

Fig.8. Pair potential normalized by $\mathrm{kT}$ (blue circles) of silica spheres ( $\mathrm{d}=1 \mu \mathrm{m}$; diameter) in water-glycerol solvent. $r$ is the distance between two particle centers. $\Phi=0.03$. 


\section{References:}

1. X. Cheng, et. al., Proc. Natl. Acad. Sci., 2012. 109 (63): p. 63-67.

2. W. Xue and G.S. Grest, Physical Review Letters, 1990.64 (4): p. 419-422.

3. Y.D. Yan, et.al., Physica A,1994. 202: p. 68-80.

4. Y.D. Yan and J.K.G. Dhont, Physica A, 1993. 198: p. 78-107.

5. J.C. Van Der Werff, et.al., Physica A, 1989. 160: p. 205-212.

6. J.C. Van Der Werff, et.al., Physica A, 1989. 160: p. 195-204.

7. R.T. Foister and T.G.M. Van de Ven. J. Fluid Mech.,1980. 96(1): p. 106-132.

8. X. Qiu, et. al., Physical Review Letters, 1988. 61(22): p. 2554-2557.

9. H. Orihara and Y. Takikawa, Physical Review E, 2011.84 (6): p. 061120(5).

10. E.C. Eckstein, et. al., J. Fluid Mech., 1977. 79(1): p. 191-208.

11. D. Leighton and A. Acrivos, J. Fluid Mech., 1987. 177: p 109-131.

12. V. Breedveld, et. al., J. Chem. Phys.,2002. 116(23): 10529-10535.

13. G. Bossis and J.F. Brady, J. Chem. Phys., 1987. 87(9): p. 5437- 5448.

14. E.S. Asmolov, Physical Review E, 2008. 77(6): p. 066312(6).

15. V. Breedveld, et. al., Physical Review E, 2001. 63(2): p. 021403(10).

16. D. Leighton and A. Acrivos, J . Fluid Mech., 1987. 181: p. 415-439.

17. Y. Wang, et. al., J. Fluid Mech., 1996. 321: p. 255-212.

18. F.R. Cunha and E.J. Hinch, J. Fluid Mech.,1996, 309: p. 211-223.

19. H.B. Eral, et.al., Langmuir, 2010. 26(22): p. 16722-16729.

20. M.I.M. Feitosa and O.N. Mesquita, Physical Review A, 1991. 44(10): p. 66776685.

21. K.V. Edmond, et. al., Physical Review E, 2012. 85(4): p. 041401(10).

22. L.P. Faucheux and A.J. Libchaber, Physical Review E, 1994. 49(6): p. 5158-5163.

23. V.N. Michailidou, et. al., Physical Review Letters, 2009. 102(6): p. 068302(4).

24. B. Cui, et. al., Physical Review Letters, 2002. 89(18): p. 188302(4).

25. M.D.C. Tinoco, et. al., Physical Review Letters, 2007. 99(13): p. 138303(4).

26. C.R. Nugent, Physical Review Letters, 2007. 99(2): p. 025702(4).

27. H. B. Eral et. al., Physical Review E., 2009. 80: p. 061403(8).

28. Y. Takikawa and H. Orihara, J. Physical Society of Japan, 2012. 81: p. 124001(4).

29. Y. Katayama and R. Terauti, Eur. J. Phys., 1996. 17: p. 136-140.

30. P.P.J.M. Schram and S.A. Trigger, Physica B., 1996. 228: p. 91-96.

31. D. Semwogerere, et. al., J. Fluid Mech., 2007. 581: p. 437-451.

32. M. Frank, et.al., J. Fluid Mech., 2003. 493: p. 363-378.

33. R.J. Phillips, et. al. Phys. Fluids A., 1992. 4 (1): p. 30-40.

34. C.J. Koh, et. al., J. Fluid Mech., 1994. 266: p. 1-32.

35. D.R. Foss and J.F. Brady, J. Fluid Mech., 1999. 401:p. 243-274.

36. D.R.Foss and J.F.Brady, J. Fluid Mech., 2000. 407: p. 167-200. 
37. X. Xu, et. al., J. Phys. Chem. Lett., 2013. 4: p. 3310-3315.

38. M. Zurita-Gotor, et. al., Physical Review Letters, 2012. 108: p. 068301 (5).

39. T. Palberg, et.al., J. Phys.: Condens. Matter, 2012. 24 :p. 464109 (19).

40. A. Morozov and D. Marenduzzo, Soft Matter, 2014. 10: p. 2748-2758.

41. K.C. Leptos, et. al., Physical Review Letters, 2009. 103: p. 198103(4).

42. J. Siepmann, et. al., J. Controlled Release, 1999. 60: p. 379-389.

43. M. Duduta, et al. Adv. Energy Mater, 2011.1: p. 511-516.

44. K. Nonaka, et. al., Materials Transactions, 2002. 43(4): p. 654-659.

45. S. Altmann, et. al., Applied Geochemistry, 2012. 27: p. 463-478.

46. M.H.G. Duits et. al., 'Measuring advection and diffusion of colloids in shear flow', submitted, 2015.

47. N.A.M. Verhaegh, and A. Vanblaaderen, Langmuir, 1994. 10(5): p. 1427-1438.

48. V.I. Uricanu and M.H.G. Duits, Langmuir, 2006. 22(18): p. 7783-7792.

49. J.C. Crocker and D.G. Grier, J. Colloid Interface Sci., 1996. 179(1): p. 298-310.

50. E. Weeks, Particle tracking codes. http://www.physics.emory.edu/ weeks/idl/index.html.

51. F. Giavazzi, et.al., Physical Review E., 2009.80: p. 031403(15).

52. R. Cerbino and V. Trappe, Physical Review Letters, 2008. 100: p. 188102(4).

53. P.J. Lu, et.al., Physical Review Letters, 2008. 108: p. 218103 (5).

54. V. A. Martinez, et.al., Biophysical Journal, 2012. 103: p. 1637-1647.

55. J. Westerweel, Meas. Sci. Technol. 1997. 8: p. 1379-1392.

56. R.J. Adrian, Annu. Rev. Fluid Mech. 1991. 23: p. 261-304.

57. G. I. Taylor, Proc. Roy. Soc. London A, 1953. 219: p. 186-203.

58. H. Bruus, Theoretical Microfluidics, 1ed. 2008: Oxford University Press.

59. X. Cheng, et. al., Science, 2011. 333: p.1276-1279.

60. S.C. Hendy, et. al., Physical Review E, 2005. 72: p. 016303(8).

61. J.P. Rothstein, Annu. Rev. Fluid Mech. 2010. 42: p. 89-109.

62. P. Joseph and P. Tabeling, Physical Review E, 2005. 71: p. 035303(4).

63. H. Brenner, Chem. Eng. Sci., 1961. 16: p. 242-251.

64. A. J. Goldman, et. al., Chem. Eng. Sci., 1967. 22: p. 653-660.

65. B. Lin, et. al., Physical Review E, 2000. 62(3): p. 3909-3919.

66. M. Bevan and D. C. Preive, J. Chem. Phys. 2000. 113: p. $1228-1236$.

67. V. N. Michailidou, et.al., J. Chem. Phys., 2013. 139: p. 164905(11).

68. Y. Han and D.G. Grier, Physical Review Letters, 2003. 91: p. 038302(4). 